\title{
Selective recall of decision-relevant information from texts
}

\author{
WALTER KINTSCH and SHERYL R. YOUNG \\ University of Colorado, Boulder, Colorado
}

\begin{abstract}
Subjects read and recalled texts of three different types: narratives, expository-descriptive texts, and expository-interference texts with high intersentence similarity. Target statements that contained some potentially decision-relevant information were embedded in these texts. Overall recall was best for the narratives, but target recall was relatively poor in this condition, presumably because the decision-relevant target sentences were perceived as being irrelevant to the macrostructure of the narratives. In the other two conditions, overall recall was lower, particularly in the interference condition, but target sentences were recalled significantly better. The recall of incidental, potentially decision-relevant information was optimal under the conditions of the present experiment when texts were only loosely structured; in tightly organized texts, such information tended to be suppressed.
\end{abstract}

What is remembered from a text depends both on the schema chosen to govern the comprehension processes and on the text itself: These two together will determine what sort of memory representation will be created, both its local and global organization. Some types of text are conventionally organized in such a way that the macrostructures (the overall interpretation of a text) that readers form are highly predictable and serve as efficient retrieval cues for the texts. This is the case with simple narratives, for which every reader brings to bear more or less the same schema, with predictable and rather statisfactory results. Other types of texts provide less efficient cues to their proper organization, and thus different readers choose somewhat different schemata and hence may obtain somewhat different interpretations of the text. Frequently, none of these fits the text perfectly, and therefore the resulting macrostructures are not well constrained by the text. As a consequence, overall recall is low for such texts. Essays and descriptive texts are often of this type.

However, if a text is recalled well because an efficient macrostructure for it has been formed, it follows that those portions of the text that are macrostructure relevant or that can be reconstructed from the macrostructure should be recalled well, whereas other portions of the text that are not directly related to the text's macrostructure would be recalled more poorly (Kintsch \& van Dijk, 1978; Singer, 1982). On the other hand, in texts that do not invite the construction of an efficient, well-defined macrostructure, information not relevant to the macrostructure might even be recalled better, in spite of the fact that overall recall for such texts is

This report is Publication No. 138 of the Institute of Cognitive Science of the University of Colorado. The authors' mailing address is: Department of Psychology, University of Colorado, Boulder, Colorado 80309. typically low; at the least, such information is not suppressed as macrostructure irrelevant.

Anderson and Pichert (1978) showed that instructions to assume a particular perspective can result in the failure to retrieve material that is irrelevant to the macrostructure formed under that special perspective. Another demonstration of how a macrostructure governs retrieval of macrorelevant and macroirrelevant items was provided by Anderson, Spiro, and Anderson (1978). These investigators had college students read a story about a couple who were either grocery shopping or dining at a restaurant. These two stories were almost identical versions of a single text. The same foods were mentioned in identical order and were associated with the same person. The results revealed that students recalled significantly more food items and attributed them to the appropriate person in the restaurant condition than in the shopping condition. Apparently, different schemata were used for the macrostructures of the two versions of the text: Who ordered what was more relevant in a restaurant situation than who got what in a grocery store.

Such considerations about memorability are of interest in the context of decision making. People frequently have to make decisions in semantically rich situations in which only some of the available information is relevant to the decision. In a previous study (Kozminsky, Kintsch, \& Boume, 1981), we investigated how readers make decisions about buying or not buying stocks on the basis of stock market reports, and what they can recall from these reports after their decision has been made. In that study, subjects read the stock market reports with the explicit goal of making a decision about the stock in question. In the present study, we investigated a situation in which people read a text that contained interspersed decision-relevant information for some purpose other than decision making. 
Kozminsky et al. showed how the decision requirements affect the way texts are read and remembered by determining the structure of the control schema that governs the comprehension process. Here, comprehension is zontrolled by a schema that is unrelated to the requirements of the decision process. What determines the likelihood that decision-relevant materials are remembered when the reading goals, as well as most of the information contained in a text, are decision irrelevant?

What decisions are made is influenced by the information that people have available in memory; factors such as recency, frequency, and saliency determine what is remembered, and thereby influence decision processes (Dellarosa \& Boume, 1982; Slovic, Fischhoff, \& Lichenstein, 1977). Thus, if subjects read a text that contains some interspersed decision-relevant information under neutral instructions and are later asked to make a decision on the basis of the material that they had read, the outcome of that decision will depend on what they can remember. More specifically, the decision will depend on whether subjects correctly recall whether the relevant information was positive or negative; they need not always remember its precise propositional content. Hence, we need to distinguish between recall of propositional content and evaluative recall. In the present study, we did not ask people to make decisions, but merely investigated what is remembered upon which a decision could be based.

Several factors that influence the ease with which a text can be recalled are known (Graesser, 1981). For instance, we know that, in general, narratives are recalled much better than are expository texts. Therefore, if a narrative contains some decision-relevant information, one would expect more of that information to be recalled than if the same information were incorporated into an expository text. However, there are some considerations that cause us to question this prediction: The very factors that are responsible for the good overall recall of a narrative ought to work against the recall of the interspersed decision-relevant information. Narratives are recalled well because readers bring to them a well-worked-out schematic structure to organize the text during comprehension and to guide retrieval at recall. This involves knowledge about human action (stories are about human actions) as well as knowledge about the conventions of storytelling. These schemata permit the reader to form a stable macrostructure that mirrors closely the course of the narrative and that supports reproduction as well as reconstruction at the time of recall. This macrostructure consists of the elements in the text that are essential for the understanding of the action sequence, that is, the plot of the story and its motivations. The incidental decisionrelevant information that was included in the story, however, is not relevant to that macrostrucure. Hence, it will not benefit from the favored status of the macrostructure in recall.
Contrast this scenario with the way an expository text would be processed. Usually, no powerful organizational schemata are available to organize such texts in a uniform way, and the reader has to work with less common, less developed knowledge sources. The outcome is a text base and macrostructure that are less predictable. Whereas one reader might concentrate on one aspect of the text, another might take a different view. Quite likely, neither organizational strategy will be as successful as in the case of a narrative for which everyone knows exactly what to do. Hence, the overall level of recall will be lower. However, although the decision-relevant material is always irrelevant in the narrative and hence not used in forming the macrostructure, this is not necessarily the case with expository texts. It is quite likely that some readers will regard these portions of the text as macrorelevant and use them to form the macrostructure. Thus, one would expect that the macro-operators of selection, generalization, and construction would be applied to this material, which would therefore be transformed and assume a dominant role in recall. Although it is not possible to predict the outcome of two opposing tendencies without a quantitative model, it would not seem unreasonable that the macrorelevance of the decision material would more than compensate for the lower overall recall in expository texts. Therefore, the decision-relevant material should be recalled as well as or better in expository texts than in narratives, in spite of the overall recall advantage of the latter.

In the present study, we used two types of expository texts. In the first (the EXPOSITORY texts), the decisionrelevant material consisted of three statements relevant to evaluating the stock of a company that was mentioned in the text, whereas the rest of the text was not concerned with the stock market but dealt with issues unrelated to it (e.g., describing the town in which the company was located). In the second type (the INTERFERENCE texts), the whole texts were written as stock market reports. Hence, all statements in these texts were highly similar to each other, and the texts were hard to read and harder to remember because of the interference generated by the high level of intersentence similarity. Thus, one would expect an even lower level of overall recall for the INTERFERENCE texts, but as far as the macrostructure is concerned, the same arguments hold for these texts as for the EXPOSITORY texts: The three decision-relevant target sentences should at least some of the time be perceived as macrorelevant and hence be transformed and recalled well.

An example of a text triplet, consisting of a narrative, a descriptive, and an interference text, each containing the same three critical target sentences (or, rather, close paraphrases, to make the texts sound more normal), is shown in the Appendix. In this example, two of the target sentences contain negative information about some fictitious company (with respect to dividends and 
capitalization), and the third contains positive information (referring to the growth of the company).

In a comparison of NARRATIVE, EXPOSITORY, and INTERFERENCE texts there ought to be significant differences in the level of overall recall for these three text types, in the order that they are listed above. As far as the recall of interspersed target statements is concerned, however, this order should be different, with poorer performance for the NARRATIVE texts and relatively better performance for the other two types. In addition, we sought to determine whether any memory differences had to do with the accessibility or the availability of information and hence employed both recall and recognition tests in our experiment.

\section{METHOD}

\section{Subjects}

Students from an introductory psychology course at the University of Colorado served as the subjects in the experiment. There were 84 subjects, divided into three groups of equal size. The subjects participated in the experiment in group sessions.

\section{Materials}

Three texts were constructed, each in three different versions (NARRATIVE, EXPOSITORY, and INTERFERENCE). The texts were 240-315 words long and consisted of four paragraphs. Three target sentences (or phrases) were embedded in each of the three texts. The target sentences were taken from the pool of stock market statements used by Kozminsky et al. (1981). The number of words varied from sentence to sentence, but each target sentence expressed three underlying propositions.

The NARRATIVE texts were brief stories about some hero, with a reasonably clear exposition-conflict-resolution structure. The target sentences were embedded in these texts in such a way that their presence was at least somewhat motivated, but they never were of crucial importance to the action. The EXPOSITORY texts were constructed in the same way, except that they were a description (of a town, a restaurant, and a factory) The INTERFERENCE texts were also descriptions, but were composed almost entirely from stock market-related statements dealing with several companies.

Each version of each text contained the same three target sentences in approximately the same locations. Specifically, there was one target sentence in each of paragraphs 1-3. Thus, there were nine target sentences in all, three for each text. Care was taken to make all texts seem as natural and coherent as possible. The three texts were General Services Company, Toothpaste Factory, and Restaurant. The NARRATIVE, EXPOSITORY, and INTERFERENCE versions of the latter are shown in the Appendix.

A $3 \times 3$ Greco-Latin square was used to counterbalance both the order of texts and the order of conditions (NARRATIVE, EXPOSITORY, and INTERFERENCE) that each subject read.

\section{Procedure}

Each subject read one version of all three texts, one in each condition (NARRATIVE, EXPOSITORY, or INTERFERENCE). Each text was printed on a separate page, and the subjects were given $2 \mathrm{~min}$ in which to read each one. They were told that they should read the texts carefully and that they would be asked questions about the texts later. After reading the three experimental texts, the subjects worked for about $20 \mathrm{~min}$ on an unrelated task that also involved reading and taking a recognition test. Thereafter, they were asked to write down everything they could recall about each text, which was identified by a short descriptive phrase. They were given $5 \mathrm{~min}$ in which to recall each text. Finally, the subjects were given a recognition test. The three target sentences from each text were each paired with a distractor, and the subjects had to select the sentence that they actually had read in the text. The distractors were obtained by making minor changes in both meaning and surface form in the target items: For instance, "The stockholders will not recieve a dividend this year" was replaced by "The stockholders have not received a dividend in years."

\section{RESULTS}

\section{Recall}

The recall protocols were scored in three ways. First, total recall was estimated by counting the total number of words in each recall protocol. Although this provided no more than a crude approximation to such measures as the total number of propositions recalled, it was quite sufficient for present purposes because total recall was of interest here only insofar as it provided a baseline for comparisons with the recall of the target sentences em. bedded in the texts. Target-sentence recall was scored in two ways: First, a propositional scoring was used, whereby each sentence was given a score from 0 to 3 . depending on how many of the three propositions of the target sentence were expressed in the protocol; second. an evaluative score was calculated for each target sen. tence-a score of 1 was assigned to the sentence if the subject stated the relevant dimension (such as dividends. earnings, capitalization, etc.) and evaluated it correctly. whether or not any of the propositions underlying that sentence were recalled correctly. For instance, if the target sentence "General Services Company doubled its earnings per share last quarter" had been recalled as "General Services Company doubled its earnings peı share this year," the score would have been 2 for prop ositional recall and 1 for evaluative recall; however, if the response had been "General Service's earnings were real good," propositional recall would have been scored as 0 , but evaluative recall as 1 . Propositional recall is essentially a measure of how much of the tar. get sentence subjects can reproduce; evaluative re. call, on the other hand, is of course tied to propositional recall (it is necessarily 1 if all propositions are recalled correctly), but it also indicates generalizations made by the subjects during or after comprehension. Thus, it reflects at least partially (especially when it is not accompanied by propositional recall) the reader's macroprocesses.

The three sets of recall scores are shown in Figure 1. Total recall of the whole text was as expected. The texts were recalled best in the NARRATIVE condition, by far worst in the INTERFERENCE condition, and inter. mediately well in the EXPOSITORY condition $[F(2,81)$ $=79.13, \mathrm{p}<.001]$. In addition, differences between texts were statistically significant (the Restaurant tex 1 was recalled better than were the other two) $[\mathrm{F}(2,162)$ $=31.77, \mathrm{p}<.001]$, and the text $x$ type interaction was 


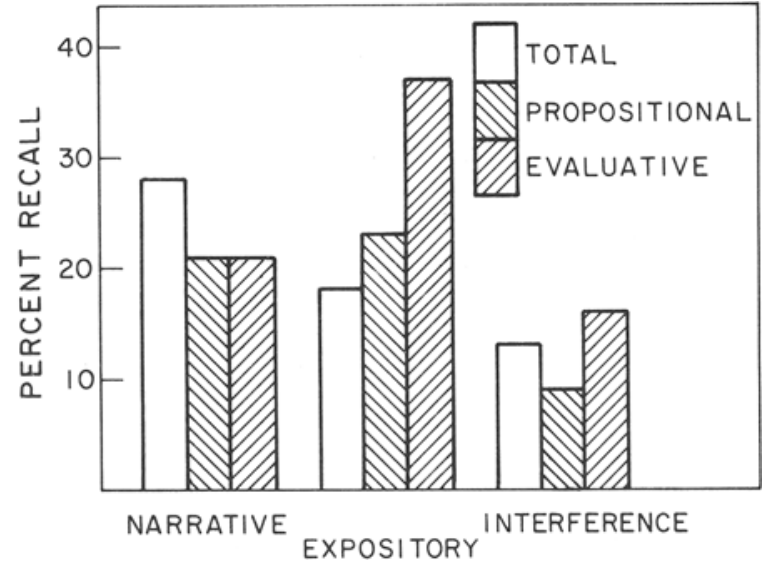

Figure 1. Three ways of scoring the recall protocols. TOTAL is the overall recall of the experimental texts; the other two scores reflect only the recall of the target sentences embedded in these texts: PROPOSITIONAL shows the percentage of propositions from the target sentences that were recalled correctly, and EVALUATIVE shows the percentage of target sentences for which the positive or negative evaluation was recalled correctly. The three experimental conditions are NARRATIVE, EXPOSITORY, and INTERFERENCE.

also significant $[F(4,162)=9.30, p<.001]$. These significant text effects show that we were not fully able to equalize the difficulty of the three texts. Since their design was entirely intuitive, this is hardly surprising. The important point here is that we did obtain substantial recall differences in the total amount of material recalled among the NARRATIVE, EXPOSITIORY, and INTERFERENCE conditions, as expected.

Propositional recall of the target sentences also differed significantly among the three conditions $[F(2,79)$ $=12.24, p<.001]$, with the NARRATIVE and EXPOSITORY conditions yielding equivalent levels of recall for the target sentences and both being superior to the INTERFERENCE condition. The texts varied between $15 \%$ and $21 \%$ recall $[\mathrm{F}(2,158)=4.78, \mathrm{p}<.01]$, but the type $x$ text interaction was not significant $[F(4,158)=$ 1.44].

The relatively better performance in the EXPOSITORY and INTERFERENCE conditions than in the NARRATIVE condition was even more pronounced in the evaluative recall scores $[F(2,79)=9.49, p<.001]$. Orthogonal comparisons revealed that evaluative recall was higher in the EXPOSITORY condition than in the NARRATIVE condition $[F(1,79)=11.85, p<.001]$ but did not differ between the NARRATIVE and INTERFERENCE conditions $(F<1)$. No significant differences in evaluative recall were observed between texts $[F(2,158)=1.24, p>.05]$ or for the text $x$ type interaction $[F(4,158)=2.36, p>.05]$.

Thus, in spite of poor overall recall, the decisionrelevant information in the target sentences in the
EXPOSITORY and INTERFERENCE conditions was recalled better than or as well as that in the NARRATIVE condition

The superiority of evaluative recall in the EXPOSITORY and INTERFERENCE conditions over that in the NARRATIVE condition derives from trials on which subjects had no propositional recall but nevertheless mentioned and evaluated correctly the relevant stock market information. This is shown in Figure 2, which displays the percentage of protocols in which both evaluative and propositional recall was observed (propositional recall was scored whenever at least one proposition from a target sentence was recalled-hence, the data are not directly comparable to those in Figure 1). It is clear from Figure 2 that, in the NARRATIVE condition, almost all evaluative recall was tied to propositional recall. However, in both types of expository texts, a substantial number of evaluative recalls occurred in the absence of any propositional recall. This differential pattern of results was statistically reliable, as determined by a chi-square test $\left[x^{2}(4)=34.32, p<.001\right]$. Thus, evaluative recall in the NARRATIVE condition was merely a by-product of reproducing the content of the story, but in the EXPOSITORY and INTERFERENCE conditions, evaluative recall occurred because readers had generalized the target sentences, abstracting the potentially decision-relevant information, and, presumably, including it in the macrostructure that they were building for these texts.

\section{Recognition}

The recognition scores were two-alternative forcedchoice data. Overall performance was $69 \%$ correct for NARRATIVE, $70 \%$ for EXPOSITORY, and $70 \%$ for

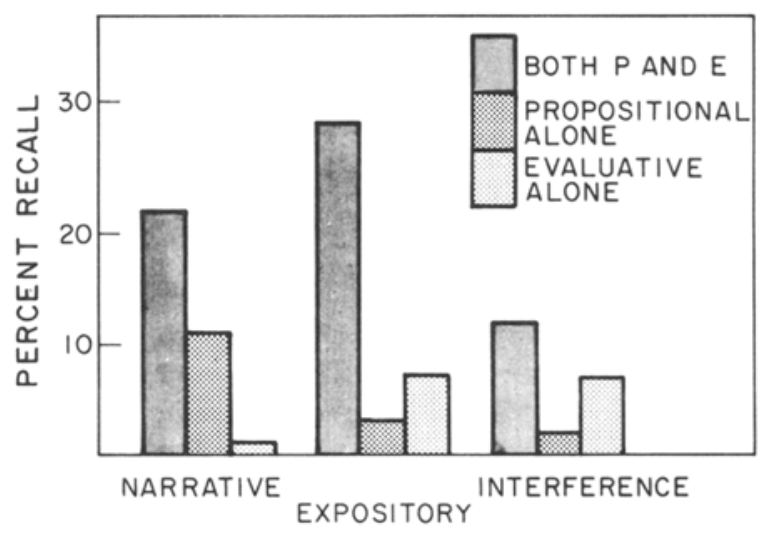

Figure 2. The relationship between propositional and evaluative recall of target sentences for the three experimental conditions: $P$ and $E$ show cases in which both (some) propositional recall of a target sentence occurred and the evaluation was reproduced correctly; PROPOSITION ALONE and EVALUATION ALONE show how of ten either propositional content of evaluations was recalled alone. 
INTERFERENCE. Thus, the observed recall differences cannot be attributed to lack of available information.

\section{DISCUSSION}

Memory and decisions based on memory (Dellarosa \& Bourne, 1982) depend on the structural properties of the texts in which the to-be-remembered information is embedded. A good, strong structure, as in a narrative, supports recall very well, whereas texts without such a structure may be much more difficult to recall. On the other hand, if we are concerned not with overall recall, but with how well people can remember interspersed decision-relevant target information that is irrelevant to the text structure, a good structure may be a hindrance rather than a benefit. Because of the very fact that the target informaton is unimportant for the text structure, it tends to be excluded from the macrostructure and will therefore be less well recalled.

These two factors may counterbalance each other, as happened in the present experiment, in which the NARRATIVE texts were recalled twice as well as the INTERFERENCE texts overall, but in which the target sentences were recalled with only a nonsignificant difference between conditions. Indeed, a decision maker would have found the EXPOSITORY condition most congenial: Significantly more evaluative recall (which is what is required for making decisions) occurred in this condition than in the other two, because it avoided, on the one hand, the extremely low overall recall levels caused by the high intersentence similarity in the IN. TERFERENCE condition and, on the other, the exclusion of the target materials from the macrostructure in the NARRATIVE condition.

Hidi, Baird, and Hildyard (1982) showed that presenting learning material in an interesting way, by including salient and entertaining anecdotes, may backfire in some situations, specifically, if it encourages readers to construct an inappropriate, misleading macrostructure. An inappropriate macrostructure was the problem here, too, in our NARRATIVE condition: Just as did Hidi, Baird, and Hildyard's anecdote, it masked, rather than highlighted, the material that was of actual concern. Of course, we are not suggesting that structure and interest be outlawedtheir motivating value may override their potentially harmful effects. But we need to understand the complex dynamics of comprehension processes if we are dealing with such problems as information analysis and pedagogical questions.

In conclusion, we note the rather interesting contrast between the recall data in Figure 1 and our recognition results. Although the context in which the target sentences appeared made a pronounced difference as far as recall was concerned, whether it was propositional or evaluative, recognition performance was equal in all three conditions. This is an interesting finding from the standpoint of memory theory, and of earlier observations in the list-learning literature in which the organi- zational structure of the to-be-remembered material was found to be a powerful determinant of recall, but not of recognition (e.g., Kintsch, 1968).

\section{REFERENCES}

Anderson, R. C., \& Pichert, J. W. (1978). Recall of previously unrecallable information following a shift in perspective. Journal of Verbal Learning and Verbal Behavior, 17, 1-12.

Anderson, R. C., Spiro, R., \& Anderson, M. C. (1978). Schemata as scaffolding for the representation of information in connected discourse. American Educational Research Journal, $15,433-440$.

Dellarosa, D., \& Bourne, L. E., Jr. (1982). Text-based decisions: Changes in the availability of facts due to instructions and the passage of time (Tech. Rep. No. 115-ONR). Boulder: University of Colorado, Institute of Cognitive Science.

Graesser, A. C. (1981). Prose comprehension beyond the word. New York: Springer.

Hidi, S., BAird, W., \& Hildyard, A. (1982). That's important but is it interesting? Two factors in text processing. In $\mathbf{A}$. Flammer \& W. Kintsch (Eds.), Discourse processing. Amsterdam: North-Holland.

KINT8CH, W. (1968). Recognition and recall of organized lists. Journal of Experimental Psychology, 78, 481-487.

KinT8Ch, W., \& van DiJK, T. A. (1978). Toward a model of text comprehension and production. Psychological Review, 85, 363394.

Kozmingxy, E., Kintsch, W., \& Bourne, L. E., Jr. (1981). Decision making with texts: Information analysis and schema acquisition. Journal of Experimental Psychology: General, 110, 363-380.

Singen, M. (1982). Comparing memory for natural and laboratory reading. Journal of Experimental Psychology: General, 111, 331-347.

Stovic, P., Fischioft, B., \& Lichtenstein, S. (1977). Behavioral decision theory. Annual Review of Psychology, 28, 1-39.

\section{APPENDIX}

Examples of NARRATIVE, EXPOSITORY, and INTERFERENCE Text, With Each Containing the Same Three Target Sentences (or Phrases, With Minor Paraphrasing) and With the Target Sentences in Italics

\section{NARRATIVE}

Bob had been trying to get a date with Lucy for weeks. He wanted to go all out, and chose to take her to the Seawitch which had opened down at the harbor in spite of the inability of its parent company Seagull, Inc., to raise sufficiently large loans last year. They arrived early and in high spirits.

Lucy seemed impressed. They ordered a glass of chablis which arrived promptly, speaking well for the service offered by the fastest growing restaurant chain in the world. However, the mood was soon spoiled, when Lucy found she could not eat the calimari she had ordered, after Bob told her it was really squid.

Bob thought she was being squeamish and foolish and resented paying all that money for nothing. He complained that the restaurant was too expensive (he didn't know that the company's stockholders will not even receive a dividend this year), and that girls in general and Lucy in particular were fickle and frivolous (he never knew how embarrassed and unhappy poor Lucy was feeling).

However, things began to look up when Lucy decided she was hungry for dessert. She ordered peach melba for two because she knew Bob liked peaches and the dessert sounded rather exotic. When the waitress brought dessert, she absentmindedly brought only one spoon. Bob was infuriated at her incompetence. However, his anger subsided when Lucy began feeding 
him with their only spoon. Bob was so pleased with the coziness that developed over dinner that he gave the waitress a ten dollar tip in spite of her incompetence.

\section{EXPOSITORY}

Seagull fast-food restaurants are constructed according to the same floor plan and decorative scheme everywhere in the world. They are designed for efficiency and cost-effectiveness, but also appeal to the romantic instincts of their clients. Had the parent company, Seagull, Inc., been able to raise sufficient loans last year, the fisherman and yacht theme of their restaurants would have been emphasized even more.

As it is, some plastic fish hanging over the counter and some of the tables, and some fairly nice but inexpensive prints of classic yacht races adorn the establishments of the fastest grow. ing restaurant chain in the world. Also, their drinks have been given cute, nautical names.

The restrooms are outfitted like small cramped ship cabins his and hers. One wonders what the stockholders who will not receive a dividend this year, might think about the restaurant decor. The carpets on the restaurant floor as well as the hallways are dark blue to enhance the maritime effect.

Also, there are thick boat lines coiled on the walls as well as an old, wooden steering wheel with protruding peg handles on the entrance door. Large fishing nets cover the ceiling. They are the kind of nets used to catch tuna. However, since it is illegal to capture dolphin along with tuna, these nets have become obsolete. That's why the Seagull chain was able to purchase them so inexpensively.

\section{INTERFERENCE}

The restaurant business is in a great deal of turmoil in these days of high interest rates. While "Lucerne," a new super-fancy French place opened up in Los Angeles backed by huge sums of venture capital, the fast food chain of Seagull, Inc., was unable to raise sufficiently large loans last year. And hamburger stands in shopping centers have made serious inroads on the traditional McDonald's operations.

Small ethnic establishments, normally family owned and operated, are springing up everywhere in the country. The Seagull chain is now the fastest growing restaurant business in the world. McDonald's, Big Boy and the like, on the other hand, have closed a third of their outlets in the depressed Northeast.

At the same time, Americans and Europeans are spending more on eating out than ever before. In some sections of California, even families with children go out more than one evening per week. Nevertheless, the stockholders of Seagull will not receive a dividend this year. The speculators backing the Lucerne expect a good return, but may be seriously disappointed in the short run.

Asian and African restaurants are opening their doors all over the country in ever greater numbers. Indian restaurants are safely established in all major cities, while more exotic cuisines such as Afghan and Ethiopian are finding more and more admirers, drawing away customers from the more familiar ethnic establishments.

(Manuscript received May 13, 1983; revision accepted for publication October 28, 1983.) 